\title{
Study of the depolarization current in mineral oils
}

\author{
Yuan Zhou, Miao Hao, George Chen \\ University of Southampton, UK \\ Yz1d10@ecs.soton.ac.uk
}

\author{
Paul Jarman, Gordon Wilson \\ National Grid UK
}

\begin{abstract}
Polarization and depolarization current measurement is a non-destructive testing method to study the dielectric properties of insulating materials. Two kinds of mineral oils with different aging time have been measured and compared. When the charge carriers drift to the electrodes, they may be blocked and accumulated in the vicinity of the electrode to form the charge layers. When the oil is discharged, the charge carriers in these charge layers will start to move backwards to the bulk and result in a depolarization current. The depolarization process can be affected by both diffusion and electric drift. In this paper, the diffusion theory has been used to study the depolarization in mineral oil and the experimental results have been fitted using exponential equations. It seems the depolarization current is mainly contributed by the diffusion of the charge carriers in the mineral oil. The assumption that there are two kinds of charge carriers in the mineral oil that made in frequency domain measurement has been verified in this paper.
\end{abstract}

Keywords - conductivity; slow charge carriers; oil; diffusion

\section{INTRODUCTION}

Polarization and depolarization are very general phenomena and they can be observed in all media except conductive media. Polarization and depolarization effects arising from the drift of charge carriers under the influence of the electric field have been well studied under both AC and DC field [1-6]. It has been found that there are fast charge carriers which can pass through the electrode and slow charge carriers can be blocked and remain in the oil [7-8]. According to our calculation, those fast charge carriers can be fully extracted in less than $0.01 \mathrm{~s}$ for a $1 \mathrm{~mm}$ thick sample when a $2 \mathrm{~V}$ voltage applied [8]. When the oil is depolarizing, these fast charge carriers can be extracted in a very short period of time, and the slow charge carriers will remain in the oil for some time. Thus, the depolarization current measurement can help us to gain a better understanding of the motion of these slow charge carriers.

Jaffe proposed a theory of polarization based on the concept that some charge carriers were unable to get through the metal electrodes [3]. Later, this theory was extended in other media and confirmed by the experimental results [1-6]. Exponential equations have been suggested to fit the curves of the depolarization current in liquid [3, 9]. Recently, an extended Debye model has been used to simulate the polarization and depolarization current in oil sample and oil/pressboard sample. The parameters that obtained from these simulations have been used for the diagnostics of insulation quality [10-12]. However, the effect of these fast charge carriers on the polarization and depolarization current has not been involved in their works.
In this paper, two kinds of mineral oils with different aging times will be measured. Exponential equations will be used to fit the experimental results. Analyses and interpretations of the experimental results will be presented in this paper. The two kinds of charge carriers assumption that made in our previous work will be verified in this paper [7].

\section{GENERAL THEORY}

When an external voltage is applied across the media, a current due to the drift of charge carriers under the electric field can be observed, this is known as the polarization. When this voltage is removed and the subject is short-circuited, the polarization that has been built up in the sample can give rise to the discharge current in an opposite direction. Jaffe provided equations with sufficient accuracy to explain the characteristics of the depolarization current [3]. However, his solutions are quite complex. If his theory is closely scrutinized, the depolarization current is only caused by the charge diffusion under the condition that the electrodes have been fully blocked. Here, a simpler model based on charge diffusion will be described.

Let us consider a parallel electrode system that filled up with the mineral oil. Assuming the charge carriers can be fully blocked by the electrode and the internal field can be treated homogeneously. As these charge carriers cannot get through the electrodes, they can only move backwards into the bulk. If the diffusion only happens in 1-D system and there are only positive charge carriers in the mineral oil, charge flows at point $x$ can be denoted as,

$$
j(x, t)=q D \frac{d n}{d x},
$$

where, $j(x, t)$ is the current density, $q$ is the charge carried by a charge carrier, $D$ is the diffusion coefficient and $n$ is the density of charge carriers. With conservation of mass, the dependence of charge density on the time can be denoted as

$$
D \frac{\partial^{2} n}{\partial x^{2}}=\frac{\partial n}{\partial t}
$$

When a DC field is applied, these charge carriers will drift to the metal electrodes. If the method of image is used here, this problem can be simplified. With this method, the charge distribution between the electrodes has been rearranged and this new distribution of charge carriers is illustrated in Fig. 1. Assuming the diffusion in a region that in the middle of these two electrodes can be neglectable, the boundary condition can be written as 


$$
n(0, t)=n(l, t)=0
$$

where $l$ is the distance between the two metal electrodes. The charge distribution at the moment that the external field is removed can be described as

$$
n(x, 0)=f(x) .
$$

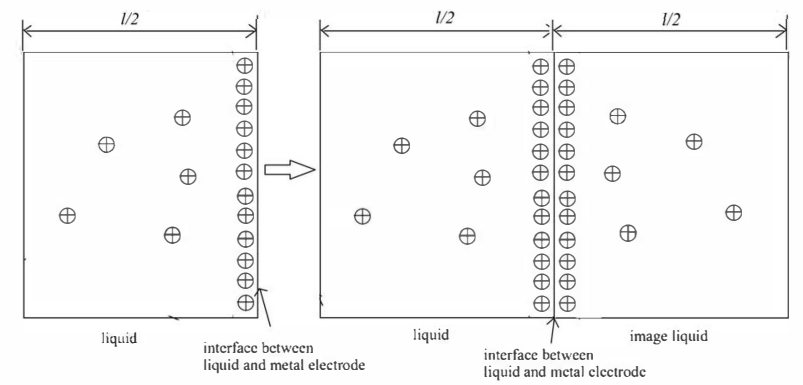

Figure 1. Image method and the distribution of charge carriers.

On considering the symmetry of the charge distribution, a solution to this 1-D diffusion equation can be denoted as

$$
Q(x, t)=\sum_{a=1}^{\infty} q \times B_{a} \times u_{a}(x, t)
$$

with

$$
\left\{\begin{array}{c}
u_{a}(x, t)=\exp \left(-\left((2 a-1) \frac{\pi}{l}\right)^{2} D t\right) \sin \left((2 a-1) \frac{\pi}{l} x\right) \\
B_{a}=\frac{1}{l} \int_{0}^{l} f(x) \sin \left((2 a-1) \frac{\pi}{l} x\right) d x
\end{array},\right.
$$

where $Q(x, t)$ is the charge density. As we are not interested in the current that is measured right after the sample is short circuited, those high order components can be ignored, thus, the charge density can be written as

$$
Q(x, t)=\left(B_{1} q\right) \exp \left(-\frac{\pi^{2} D}{l^{2}} t\right) \sin \left(\frac{\pi}{l} x\right) .
$$

If the majority of the charge carriers are in the charge layer which is close to the electrode and the total charge in that layer is $Q_{1}, B_{1}$ can be approximated as

$$
B_{1} \approx 2 Q_{1} /(l q) .
$$

With conservation of electric charge

$$
\frac{\partial Q(x, t)}{\partial t}=\frac{\partial j(x, t)}{\partial x}
$$

the current density can be denoted as

$$
j(x, t)=\frac{2 \pi D Q_{1}}{l^{2}} \exp \left(-\frac{\pi^{2} D}{l^{2}} t\right)\left(\left|\cos \left(\frac{\pi}{l} x\right)\right|-1\right) .
$$

The total current that is measured by an am-meter can be integrated as

$$
J_{+}(t)=\frac{2}{l} \int_{0}^{l / 2} j(x, t) d x=-\frac{2(\pi-2) Q_{1} D}{l^{2}} \exp \left(-\frac{\pi^{2} D}{l^{2}} t\right)
$$

If there are multiple kinds of charge carriers, this current can be written as

$$
J_{+}(t)=-\sum_{a=1}^{n} \frac{2(\pi-2) Q_{a} D_{a}}{l^{2}} \exp \left(-\frac{\pi^{2} D_{a}}{l^{2}} t\right),
$$

in which, $Q_{a}$ and $D_{a}$ are the total charge in the charge layer and the diffusion coefficient for the $a$-th charge carriers. For negative charge carriers, we can obtain

$$
J_{-}(t)=\sum_{a=1}^{n} \frac{2(\pi-2) Q_{a} D_{a}}{l^{2}} \exp \left(-\frac{\pi^{2} D_{a}}{l^{2}} t\right) .
$$

Here, we assume that the diffusion coefficient, mobility and number density of positive ions are equal to those of negative ions. The depolarization current that is contributed by both positive and negative charge carriers can be written as

$$
J_{d e p}(t)=J_{+}(t)+J_{-}(t)=-\sum_{a=1}^{n} \frac{4(\pi-2)\left|Q_{a}\right| D_{a}}{l^{2}} \exp \left(-\frac{\pi^{2} D_{a}}{l^{2}} t\right)(
$$

As seen from Eq. (12) and (13), their exponential terms are similar to that derived by Jaffe and Adamczewski $[3,9]$.

\section{COMPARISON BETWEEN EXPERIMENT AND THEORY}

The electrode system for experiment will use the experimental kit that was initially designed by Kuchler [13]. This electrode system will be placed in a sealed glass test cell. The distance between the high voltage electrode and the measuring electrode is $1 \mathrm{~mm}$, the gap between the measuring electrode and guard electrode is $0.5 \mathrm{~mm}$ and the edge radiuses of the measuring electrode and the guard electrode are $0.5 \mathrm{~mm}$. All the electrodes are made of stainless steel. The test cell will be vacuumed first and then injected with the mineral oil from a vacuum vessel to avoid contamination from the air. The test will be performed at different temperatures $\left(30{ }^{\circ} \mathrm{C}\right.$ and $\left.90{ }^{\circ} \mathrm{C}\right)$ and different electric fields $(1 \mathrm{kV} / \mathrm{mm}, 2 \mathrm{kV} / \mathrm{mm}$ and 5 $\mathrm{kV} / \mathrm{mm}$ ). Two kinds of mineral oils will be tested, the fresh mineral oil (oil A), the aged mineral oil that has been aged for 10 years (oil B). A DC voltage is applied across the test object for 3 hours. When the voltage is removed, the object will be short-circuited and a depolarization current will be recorded.

As seen from Eq. (14), the curve for depolarization current can be calculated by a formula of the type

$$
i=-\sum_{i=1}^{n} C_{i} \exp \left(-b_{i} t\right)
$$

Figures 2-5 represent the comparison between theory and measurements on the depolarization current in oil A and oil B. The constant used in this calculation are given in Table I. It has been seen that the theoretical curves represent the observed data with good accuracy.

With Eq. (14), the charge in the charge layers and the mobility of the charge carriers can be denoted as

$$
\left\{\begin{array}{c}
Q_{i}=\pi^{2} C_{i} /\left[4(\pi-2) b_{i}\right] \\
\mu_{i}=b_{i} q l^{2} /\left(k T \pi^{2}\right)
\end{array}\right.
$$


TABLE I.

CONSTANTS USED IN THE CALCULATION OF FigURES. 2-5

\begin{tabular}{|c|c|c|c|c|c|c|c|c|}
\hline & \multirow{2}{*}{\multicolumn{2}{|c|}{$C_{i}$}} & \multicolumn{3}{|c|}{$30^{\circ} \mathrm{C}$} & \multicolumn{3}{|c|}{$90^{\circ} \mathrm{C}$} \\
\hline \multirow{4}{*}{ Oil A } & & & 1 & $2 \times 10^{-2}$ & $1 \times 10^{-3}$ & $1 \times 10^{-1}$ & $2 \times 10^{-2}$ & $2 \times 10^{-3}$ \\
\hline & \multirow{3}{*}{$b_{i}$} & $1000 \mathrm{~V} / \mathrm{mm}$ & $2.5 \times 10^{-11}$ & $1.6 \times 10^{-11}$ & $1.7 \times 10^{-12}$ & $1.9 \times 10^{-10}$ & $1.9 \times 10^{-10}$ & $2.0 \times 10^{-12}$ \\
\hline & & $2000 \mathrm{~V} / \mathrm{mm}$ & $6.0 \times 10^{-11}$ & $3.3 \times 10^{-11}$ & $2.9 \times 10^{-12}$ & $1.0 \times 10^{-10}$ & $2.6 \times 10^{-10}$ & $4.0 \times 10^{-12}$ \\
\hline & & $5000 \mathrm{~V} / \mathrm{mm}$ & $5.0 \times 10^{-11}$ & $8.7 \times 10^{-11}$ & $7.2 \times 10^{-12}$ & $6.0 \times 10^{-10}$ & $3.6 \times 10^{-10}$ & $5.0 \times 10^{-12}$ \\
\hline \multirow{4}{*}{ Oil B } & & $C_{i}$ & 1 & $5 \times 10-2$ & $4 \times 10^{-4}$ & $2 \times 10^{-1}$ & $2 \times 10^{-2}$ & $7 \times 10^{-4}$ \\
\hline & \multirow{3}{*}{$b_{i}$} & $1000 \mathrm{~V} / \mathrm{mm}$ & $1.5 \times 10^{-11}$ & $1.0 \times 10^{-11}$ & $1.4 \times 10^{-12}$ & $1.5 \times 10^{-10}$ & $1.6 \times 10^{-10}$ & $2.2 \times 10^{-11}$ \\
\hline & & $2000 \mathrm{~V} / \mathrm{mm}$ & $4.0 \times 10^{-11}$ & $1.8 \times 10^{-11}$ & $2.5 \times 10^{-12}$ & $3.9 \times 10^{-10}$ & $3.0 \times 10^{-10}$ & $2.3 \times 10^{-11}$ \\
\hline & & $5000 \mathrm{~V} / \mathrm{mm}$ & $1.9 \times 10^{-10}$ & $5.0 \times 10^{-11}$ & $5.4 \times 10^{-12}$ & $8.0 \times 10^{-10}$ & $4.5 \times 10^{-10}$ & $2.9 \times 10^{-11}$ \\
\hline
\end{tabular}

Here, we assume that the diffusion coefficient, mobility and number density of positive ions are equal to those of negative ions. Therefore, the total charge in the charge layer in which the positive charge carriers accumulate can be denoted as

$$
Q_{t o t}=\left(\sum_{i} Q_{i}\right) / 2 \text {. }
$$

These total charges for oil A and oil B can be found in Table II. It seems that the amount of charge in charge layer that is close to electrode will increase with aging and temperature.

TABLE II. TOTAL CHARGES FOR OIL A AND OIL B

\begin{tabular}{|c|c|c|}
\hline $\mathbf{Q}_{\text {tot }}$ & $30^{\circ} \mathrm{C}$ & $90^{\circ} \mathrm{C}$ \\
\hline Oil A & $2.8 \times 10^{-9} \mathrm{C}$ & $1.4 \times 10^{-8} \mathrm{C}$ \\
\hline Oil B & $4.1 \times 10^{-9} \mathrm{C}$ & $4.4 \times 10^{-8} \mathrm{C}$ \\
\hline
\end{tabular}

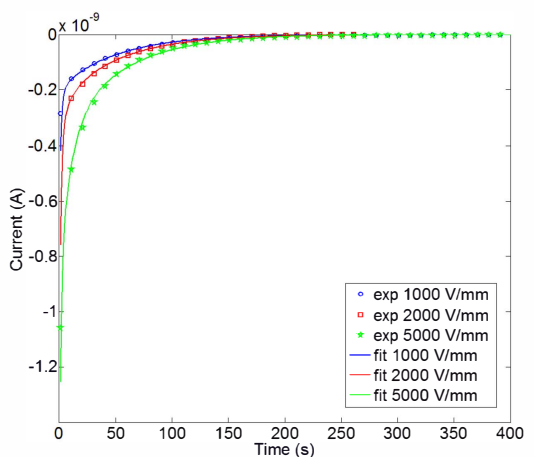

Figure 2. Comparison between theory and measurements on the depolarization current in oil $\mathrm{A}$ at $90^{\circ} \mathrm{C}$. The drawn-out curves are theoretical value from Eq. (15).

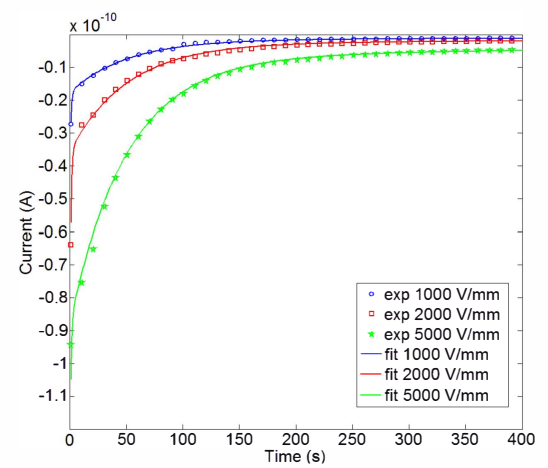

Figure 3. Comparison between theory and measurements on the depolarization current in oil A at $30^{\circ} \mathrm{C}$. The drawn-out curves are theoretical value from Eq. (15).

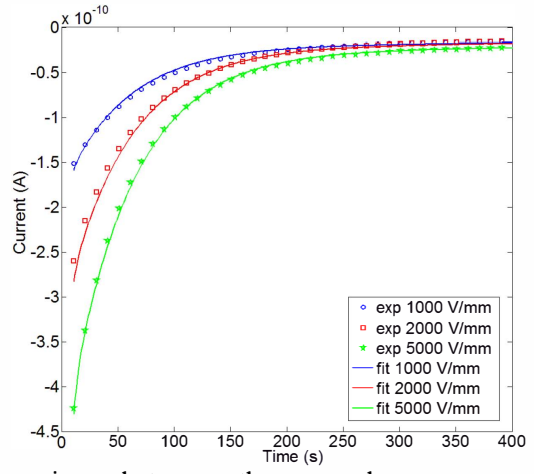

Figure 4. Comparison between theory and measurements on the depolarization current in oil $\mathrm{B}$ at $90^{\circ} \mathrm{C}$. The drawn-out curves are theoretical value from Eq. (15).

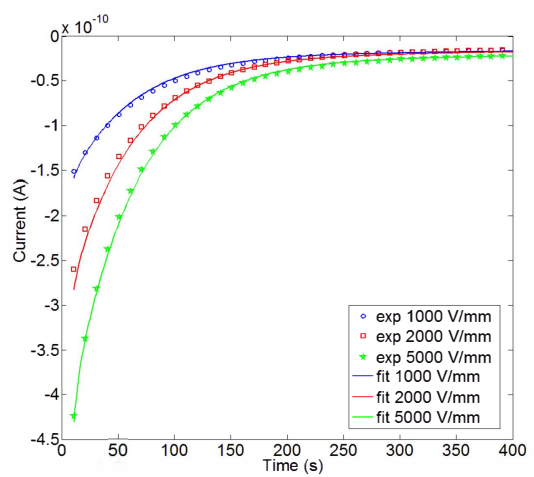

Figure 5. Comparison between theory and measurements on the depolarization current in oil $\mathrm{B}$ at $30^{\circ} \mathrm{C}$. The drawn-out curves are theoretical value from Eq. (15).

In our previous work, a model with two types of charge carriers has been proposed. The first kind of charge carriers can be dissociated in the bulk and can be blocked by the electrode, while the second kind of charge carriers are charged in the vicinity of the electrode and can be neutralized when they get close to the opposite electrode, which is also known as the injected charge carriers [7]. As the mobility for the second kind should be several orders of magnitude higher than that for the first kind, these injected charge carriers can be extracted in a very short of time after the sample has been short-circuited [8]. Thus, the motions of the first kind of charge carriers are crucial to the depolarization process. For a completely blocked electrode, the maximum charge due to the $i$-th positive charge carriers of the first kind, $Q_{i \max }$, that can be accumulated in the charge layer can be estimated using the following equation

$$
Q_{i \max }=S l \sigma_{i} /\left(2 \mu_{i}\right)+\sigma_{i}^{2} t_{p o l} S l /\left(2 \mu_{i} \varepsilon_{r}\right)=Q_{i} / 2,
$$


in which, $S$ is the surface area of the measuring electrode, $\mu_{\min }$ is the slowest mobility of the charge carriers in the oil, $\sigma_{i}$ is the conductivity that is contributed by the motion of the $i$-th positive and negative charge carriers, $t_{p o l}$ is the polarization time and $\varepsilon_{r}$ is the permittivity of the oil. The first term in Eq. (18) comes from the initial charge distribution in oil when there is no field present. The second term is related to the newly generated charge carriers due to the dissociation.

Here, we define the ratio of the conductivity contributed by the first kind of charge carriers as

$$
\beta=\left(\sum_{i} \sigma_{i}\right) / \sigma
$$

in which, $\sigma$ is the conductivity that is measured according to the procedure defined in IEC 61620 [14].

By calculating this maximum charge with Eq. (18) providing $S=3.63 \times 10^{-3} \mathrm{~m}^{2}, \quad t_{p o l}=1 \times 10^{4} \mathrm{~s} \quad$ and $\varepsilon_{r}=1.95 \times 10^{-11}$, we obtain $\beta$ for both oil $\mathrm{A}$ and oil $\mathrm{B}$ under a field of $1000 \mathrm{~V} / \mathrm{mm}$. The dependence of $\beta$ on different temperatures are given in Table III.

TABLE III. COEFFICIENT OIL $\beta$ FOR OIL A AND OIL B

\begin{tabular}{|c|c|c|c|}
\hline \multicolumn{2}{|c|}{$\beta$} & $30^{\circ} \mathrm{C}$ & $90^{\circ} \mathrm{C}$ \\
\hline \multirow{2}{*}{$\begin{array}{c}\text { Time - } \\
\text { domain }\end{array}$} & Oil A & 1.54 & 0.43 \\
\hline & Oil B & 0.06 & 0.01 \\
\hline \multirow{2}{*}{$\begin{array}{l}\text { Frequency- } \\
\text { domain }\end{array}$} & Oil A & 0.9 & 0.45 \\
\hline & Oil B & 0.4 & 0.065 \\
\hline
\end{tabular}

As seen from Table III, the parameter $\beta$ for oil A is higher than that for oil $\mathrm{B}$ and it decreases with the temperature. The parameter $\beta$ calculated from time domain measurement share the same tendency with that obtained based on the frequency domain method. It is rather surprising that the theoretical conductivity that is contributed by the first kind of charge carriers is much higher than the initial conductivity. As the dissociation rate and ionic injection rate increase with the field and the initial conductivity is measured under a low electric field $(10 \mathrm{~V} / \mathrm{mm})$, there should be more charge carriers in the oil when field is $1000 \mathrm{~V} / \mathrm{mm}$ [15-18]. Also, this coefficient $\beta$ for oil B is slightly lower than that obtained from the frequency domain method. Please note that one of our assumptions is the electrodes are completely blocked. But in real case, there is a chance that the charge carriers can be neutralized when they get close to the electrode. Jaffe has described this extraction process and a high extraction rate can be attained if the total charge in the charge layer is really large [4]. Since the conductivity of oil $\mathrm{B}$ is about 20 30 times higher of oil A, more charge carriers will drift towards the electrodes and make the charge density near electrodes really high in oil $\mathrm{B}$, therefore, the extraction rate should be taken into consideration when the oil has a high conductivity.

To sum up, the depolarization current is fitted using exponential equations. the parameter $\beta$ that calculated from the diffusion model decreases with aging or temperature. It seems the assumption of the existence of the fast charge carriers is valid in the analysing of the depolarization current.

\section{CONCLUSION}

The time dependent curves of depolarization current of the mineral oil can be well fitted using the exponential equations. The total density in the charge layer increases with temperature and aging of the oil. The ratio of the conductivity contributed by the motion of the first kind of charge carriers over the total conductivity, $\beta$, decrease with temperature and aging of the mineral oil.

\section{ACKNOWLEDGMENT}

The authors are grateful to National Grid UK for their financial support.

\section{REFERENCES}

[1] G Jaffé, "Theory of Conductivity of Semiconductors", Phys. Rev, Vol. 85, 354-363, 1952

[2] H. C. Chang and G. Jaffé, "Polarization in Electrolytic Solutions. Part I. Theory", J. Chem. Phys. Vol. 20, 1071-1087, 1952

[3] G. Jaffé and C. Z. LeMay, "On Polarization in Liquid Dielectrics", J. Chem. Phys. Vol. 21, 920-928, 1953

[4] G. Jaffé and J. A. Rider "Polarization in Electrolytic Solutions. Part II. Measurements" J. Chem. Phys. 20, 10771952

[5] J. R. Macdonald, "Theory of ac Space-Charge Polarization Effects in Photoconductors, Semiconductors, and Electrolytes" Phys. Rev. Vol. 92, 4-17 1953

[6] J. Gavis, "Electrical Conductivity of Low Dielectric Constant Liquids by dc Measurement" J. Chem. Phys. Vol. 41, 3787-3793 1964

[7] Y Zhou, M Hao, G Chen, G Wilson and P Jarman "Space charge Polarization in Insulating Mineral Oil" Proc. IEEE CEIDP, Shenzhen, China, Oct, 2013, vol 1, p.587

[8] Y Zhou, M Hao, G Chen, G Wilson and P Jarman "Study of the dielectric response in mineral oil using frequency-domain measurement" J. Appl. Phys. 1151241052014

[9] I. Adamczewski, Ionization, Conductivity and Breakdown in Dielectric Liquids, Taylor and Francis, 1969.

[10] T. K. Saha and P. Purkait, "Investigation of polarization and depolarization current measurements for the assessment of oil-paper insulation of aged transformers," Dielectrics and Electrical Insulation, IEEE Transactions on, vol. 11, pp. 144-154, 2004

[11] N. A. Muhamad, et al., "Polarization and Depolarization Current (PDC) tests on biodegradable and mineral transformer oils at different moisture levels," in Power Engineering Conference, 2009. AUPEC 2009. Australasian Universities, 2009, pp. 1-6

[12] W. Zaengl, "Dielectric spectroscopy in time and frequency domain for HV power equipment. I. Theoretical considerations", IEEE Electrical Insul. Mag., Vol. 19, No. 5 ,Sep-Oct 2003, p. 5-19

[13] A. Küchler, M. Liebschner, A. Reumann, C. Krause, U. Piovan, B. Heinrich, R. Fritsche, J. Hoppe, A. Langens and J. Titze "Evaluation of Conductivities and Dielectric Properties for Highly Stressed HVDC Insulating Materials" CIGRE Session 2010, Paper D1_106_2010,

[14] IEC 61620 Insulating Liquids -Determination of the Dielectric Dissipation Factor By Measurement Of The Conductance And Capacitance - Test Method First Edition 1998-11

[15] N.Felici J.P.Gosse "Injection d'ions par des électrodes métalliques dans les hydrocarbures liquides de résistivité élevée" Rever. Phys. Appl., 14, 1979

[16] L.Onsager "Deviations From Ohm's Law In Weak Electrolytes" J. Chem. Phys., Vol. 2, 599-615. 1934 